\title{
Polyindole Based Nickel-Zinc Oxide Nanocomposite - Characterization and Antifungal Studies
}

\author{
Dedhila Devadathan and R. Raveendran
}

\begin{abstract}
In the present work polyindole based nickel-zinc oxide nanocomposite was prepared. Here nickel-zinc oxide was prepared separately in presence of capping agent using well known co-precipitation method. Polyindole based nickel-zinc oxide nanocomposite was prepared using chemical oxidation method. Polyindole and nickel-zinc oxide was also prepared separately to compare their properties with their counterparts. The samples have been analysed using SEM, FTIR, XRD techniques. Polyindole showed a cauliflower like pattern in SEM picture and the surface morphology was changed entirely in the case of nanocomposite. FTIR showed the vibration bands of both polyindole and nickel- zinc oxide in the nanocomposite. From the XRD spectrum the particle size was calculated using Scherrer's equation. The dielectric properties of these materials were studied and the dependence of dielectric constant and a.c conductivity with frequency of these samples were analysed. Antifungal activity of the nanocomposite and their counterparts were studied separately on the fungi Penicillium Chrysogenum. The nanocomposite showed activity of $1 \mathrm{~cm}$, nickel - zinc oxide showed $0.7 \mathrm{~cm}$ activity, whereas polyindole, nickel oxide and zinc oxide showed no activity.
\end{abstract}

Index Terms - Chemical co-precipitation, chemical oxidation, conducting polymer, nanocomposite, polyindole.

\section{INTRODUCTION}

In the large field of nanotechnology, conducting polymer matrix based nanocomposites have become a prominent area of current research and development. Conducting polymers have electrical and electrochemical properties that show great promise for commercial application in rechargeable batteries, Schottky diodes, light emitting diodes, transistors, etc. There has been increasing attention paid to the application of conducting polymers in various fields of electronics, industry and agriculture. In recent years, the study of the electroactive properties of heterocyclic conducting polymers containing nitrogen atoms like polyaniline, polypyrrole, polycarbazole and their substituted derivatives had attracted considerable interest. However, among the various aromatic compound based conducting polymers, polyindole and its derivatives had only scarcely been investigated, although there exists close structural similarities with the polymers mentioned above. The oxidative coupling of indole can be obtained either by chemical or electrochemical techniques [1], [2].

The metal oxides are also an extremely important technological materials for use in electronic and photonic devices, in chemical industries and in medical fields [3]-[6]. An expanding trend for the nanomaterials is the fabrication

Manscript received November 19, 2013; revised January 14, 2014.

The authors are with Nanoscience Research Laboratory, Department of Physics, Sree Narayana College, Kollam, Kerala, India (email: dedhila@yahoo.com). of composite structures and devices with materials capable of enhancing the properties of the composite material. In the present study polyindole based nickel-zinc oxide nanocomposite was prepared and analyzed.

\section{MATERIALS AND MeTHODS}

AR grade chemicals obtained from Merck were used for the preparation of nano oxides, polymer and nanocomposites. Both the individual nano oxides and nickel-zinc oxide were prepared by the co- precipitation method in presence of capping agent. Polyindole and polyindole based nickel-zinc oxide nanocomposite were prepared using chemical oxidation method. Polyindole and nickel-zinc oxide were prepared to compare their properties with their counterparts. $\mathrm{NiO}, \mathrm{ZnO}$ and nickel-zinc oxide nanocomposite annealed at $500^{\circ} \mathrm{c}$ for three hours and polyindole \& polyindole based nanocomposite in the as prepared form were used for analysis. For the preparation of the nanocomposite $500^{\circ} \mathrm{c}$ heated nickel-zinc oxide was used.

The surface morphology of the powdered sample was obtained by scanning electron microscope (SEM) [JEOL/EO JSM-6390]. FTIR studies were made using Perkin-Elmer FTIR Spectro Photo Meter in the wavenumber range 400 $\mathrm{cm}^{-1}$ and $4000 \mathrm{~cm}^{-1}$ by $\mathrm{KBr}$ disc method. XRD study was carried out using XPERT-PRO model powder diffractometer (PAN analytical, Netherlands) employing $\mathrm{Cu}-\mathrm{K}_{\alpha}$ radiation $\left(\lambda=1.54060 \mathrm{~A}^{\circ}\right)$ operating at $40 \mathrm{kV}, 30 \mathrm{~mA}$. Dielectric were done by using KIOKI 3552 LCR meter operated at base voltage $0.01 \mathrm{~V}$.

\section{RESULTS AND CONCLUSIONS}

\section{A. SEM Analysis}

From SEM analysis it was found that the morphology of $\mathrm{NiO} \& \mathrm{ZnO}$ was changed when they were in composite state (Fig. 1(a) to (c). Polyindole showed a cauliflower like pattern which was changed entirely in the case of nanocomposite, Fig. 1 (d) \& (e).

\section{B. FTIR Analysis}

A close examination of the FTIR spectra showed that the precursor exhibits the basic absorption frequency bands between 4000 and $400 \mathrm{~cm}^{-1}$. The prominent bands observed in the spectrum for both oxides (Fig. 2(a)) includes: 3430 $\mathrm{cm}^{-1}$ due to hydrogen-bonded hydroxyl groups, $1630 \mathrm{~cm}^{-1}$ due to bending vibration of water molecules. The weak bands at $421 \mathrm{~cm}^{-1}$ for $\mathrm{NiO}$ and $436 \mathrm{~cm}^{-1}$ for $\mathrm{ZnO}$ were attributed to metal oxide vibrations. Nickel-zinc oxide also showed the characteristic peaks. In the case of polyindole (Fig. 2(b)) the sharp band expected at about $3200 \mathrm{~cm}^{-1}$ for 


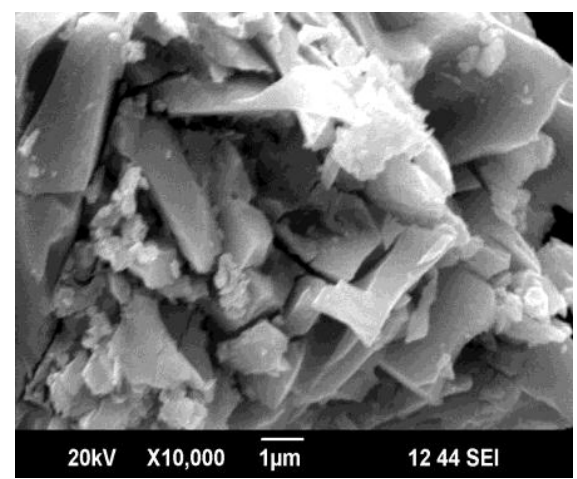

(a)

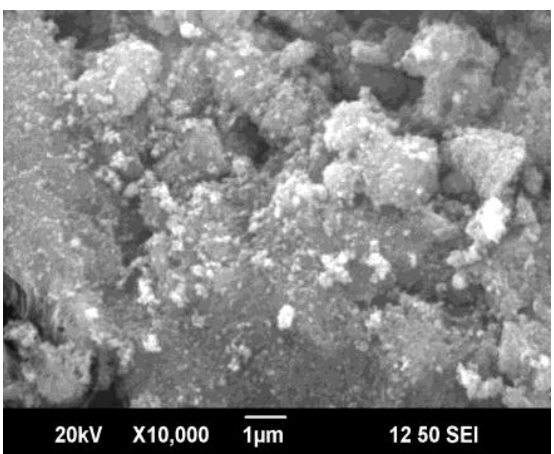

(b)

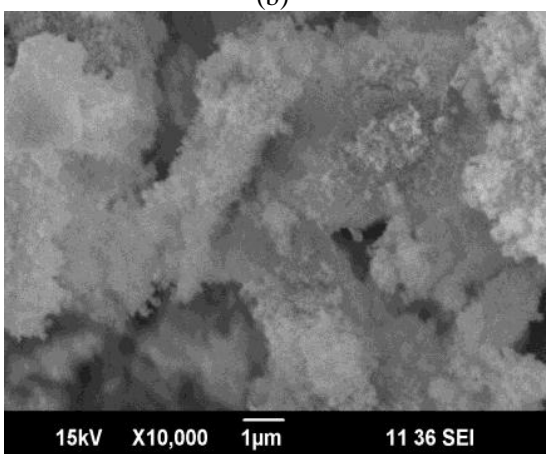

(c)

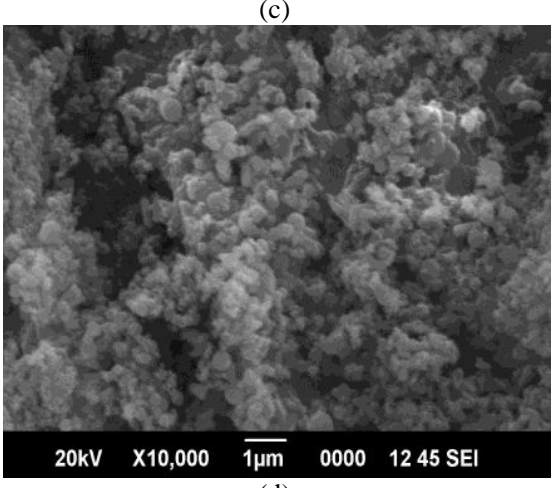

(d)

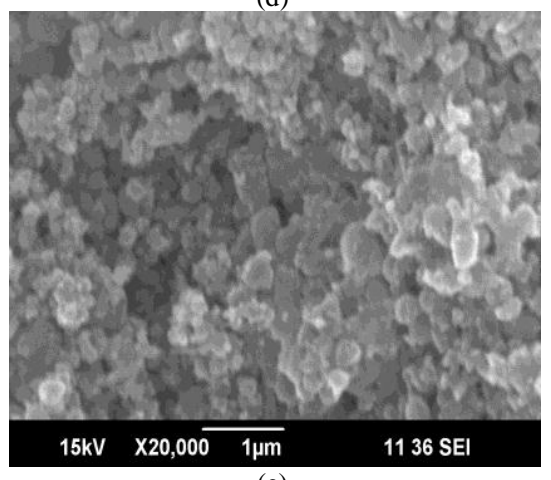

(e)

Fig. 1. SEM images of (a) Nickel oxide (b) Zinc oxide (c) Nickel-Zinc oxide (d) Polyindole (e) Polyindole Nickel-Zinc nanocomposite. the bonded N-H stretch is absent, indicating the participation of nitrogen in the polymerization. Peaks at $1458 \mathrm{~cm}^{-1}$ are induced by aromatic alkene, $1331 \mathrm{~cm}^{-1}$ is due to $\mathrm{C}-\mathrm{N}$ vibration and $744 \mathrm{~cm}^{-1}$ is due to the bending mode of aromatic alkene. The weak peak at $1105 \mathrm{~cm}^{-1}$ corresponds to oxidized form of polyindole. It is inferred that the chloride ions from the oxidant serve as counter ions for oxidized polyindole. The IR spectrum of polyindole based nickel-zinc oxide nanocomposite shows all the characteristic vibrations of polyindole. The characteristic band for nickel-zinc oxide stretching at $457 \mathrm{~cm}^{-1}$ is also observed.

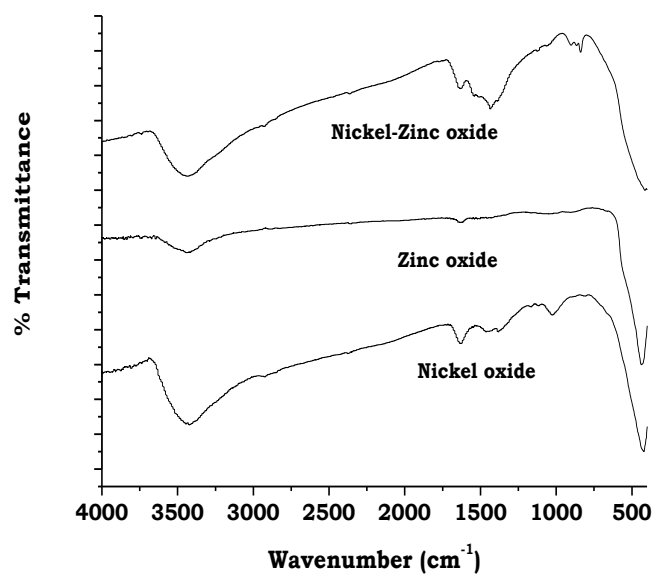

(a)

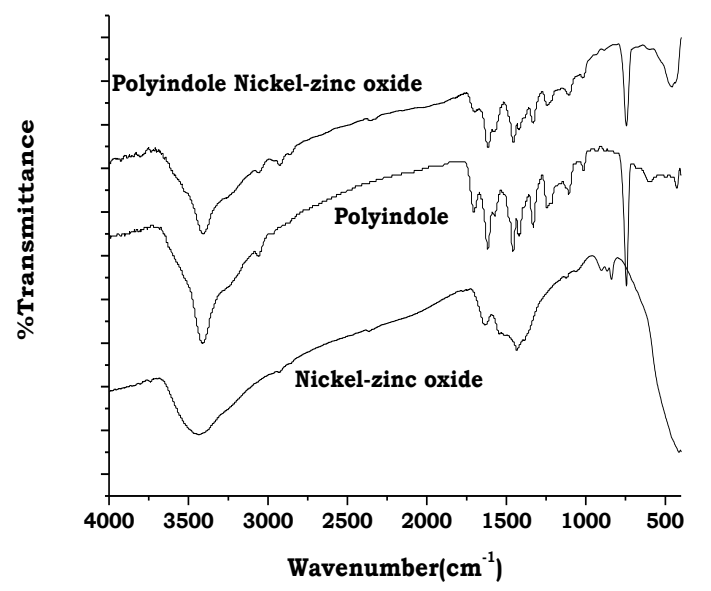

(b)

Fig. 2. FTIR spectrum.

\section{XRD Spectrum Analysis}

XRD analysis of all the samples was done. From the XRD spectra, the particle size was calculated using Scherrer equation,

$$
D=k \lambda /\left(\beta_{h k l}\right) \text { measured } \times \cos \theta_{h k l}
$$

Here, $D$ is the average crystallite size normal to the reflecting planes, $k$ - is the shape factor which lies between 0.95 and 1.15 depending on the shape of the grains $(k=1$ for spherical crystallites), $\lambda$-is the wavelength of $x$-ray used and $\left(\beta_{h k l}\right)_{\text {measured }}$ is the FWHM of the diffraction line in radians and $\theta_{\mathrm{hkl}}$ is the Bragg angle corresponding to the diffraction line arising from the planes designated by Miller indices $(h k l)$. The particle size for all the samples are in the range 20 $-40 \mathrm{~nm}$. 
XRD spectrums were matched with JCPDS cards. The XRD's of the annealed zinc oxide was found to be in matching with the JCPDS card no. \#79-0205, according to which $\mathrm{ZnO}$ is a hexagonal system with primitive lattice. The lattice constants $(a=3.235$ and $c=5.187)$ calculated also matched with JCPDS value. The XRD data of nickel oxide was in matching with the JCPDS card no. \#78-0423, according to which $\mathrm{NiO}$ is a cubic system with fcc lattice and the calculated value of $a=4.213$. Nickel-zinc oxide was in matching with JCPDS card no. \# 75-0270, according to which the nickel- zinc oxide was a cubic system with fcc lattice and the calculated value of $a=4.188$. From Fig. 3(b) $\&$ (c) it is clear that the amorphous nature of the polymer was modified in the presence of nickel-zinc oxide nanocomposite.

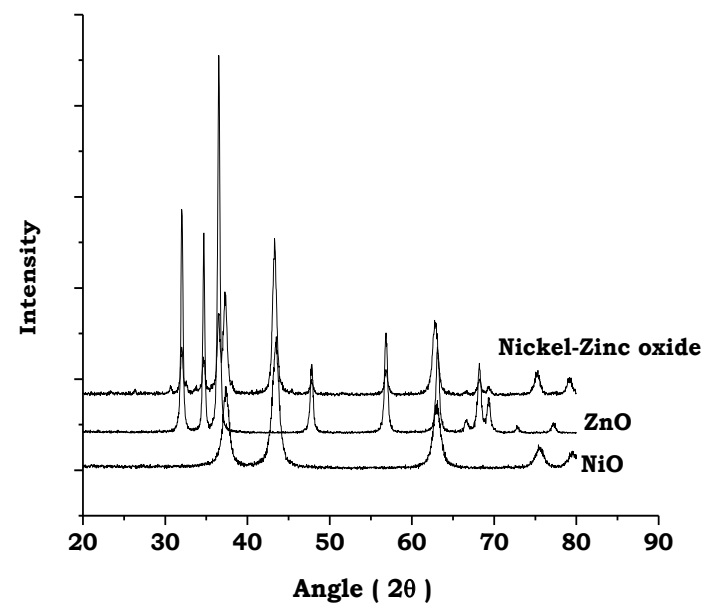

(a)

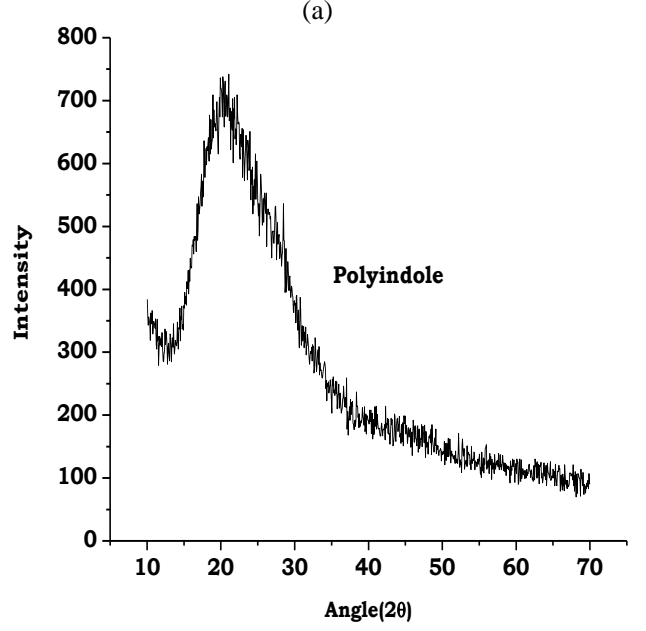

(b)

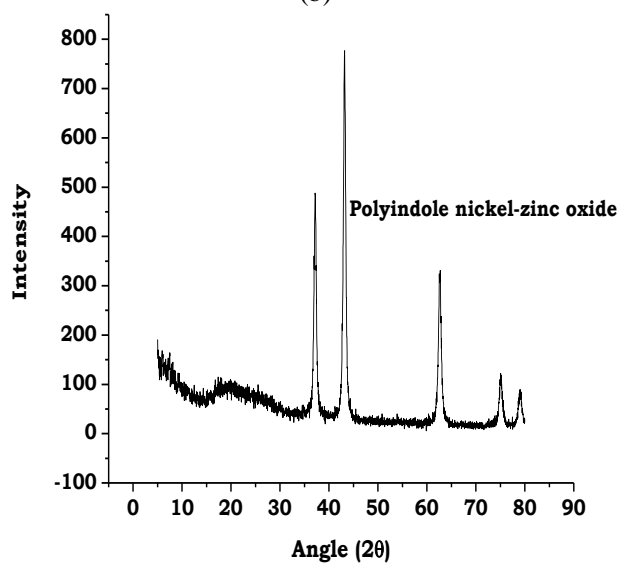

(c)

Fig. 3. XRD spectrum.

\section{Dielectric Studies}

The dielectric properties of these materials were studied (Fig. 4) and the dependence of, capacitance, loss factor and impedance with frequency of these samples were analysed. The capacitance of the polyindole based nickel-zinc oxide nanocomposite was found to be greater than its counter parts. The impedance of these materials was found to be in reverse order which was in agreement with the theory. From this study it is concluded that with proper tuning the polyindole based nickel-zinc oxide nanocomposite can be used as a good capacitor.

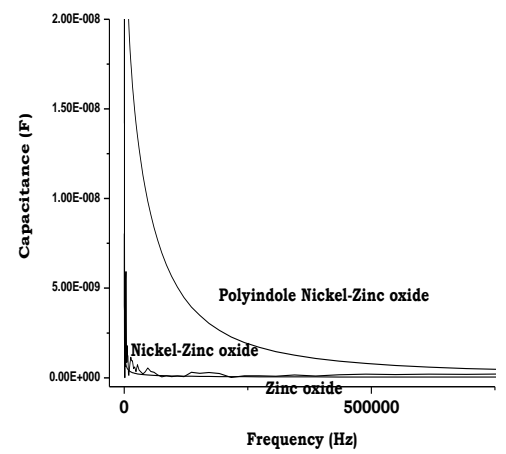

(a)

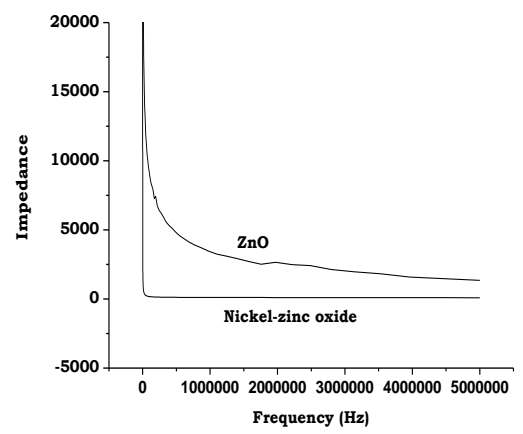

(b)

Fig. 4. Dielectric studies.

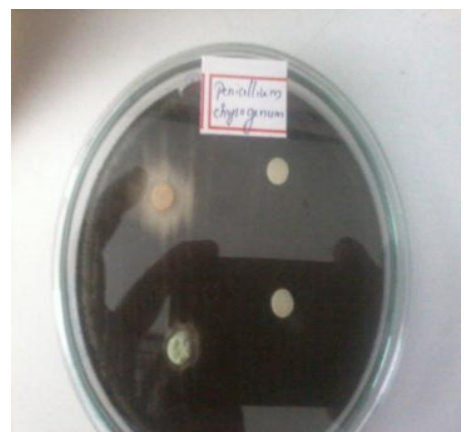

Fig. 5. Antifungal studies of all the samples on the fungi Penicillium Chrysogenum.

\section{E. Antifungal Studies}

Antifungal activity of the nanocomposite and their counterparts were studied separately on the fungi Penicillium Chrysogenum. The Polyindole based nickel-zinc oxide nanocomposite showed activity of $1 \mathrm{~cm}$, nickel-zinc oxide showed $0.7 \mathrm{~cm}$ activity, whereas polyindole, nickel oxide and zinc oxide showed no activity. From this study it could be concluded that the nanocomposite could be used as an antifungal material for the fungi Penicillium Chrysogenum. Fig. 5 below, top left shows the activity of the nanocomposite, bottom left shows the activity of nickelzinc oxide and the right top and right bottom shows that of 
nickel oxide and that of zinc oxide which have no activity.

\section{CONCLUSIONS}

In the present work polyindole based nickel - zinc oxide nanocomposite was prepared. Here nickel - zinc oxide was prepared separately in presence of capping agent using well known co-precipitation method. Polyindole based nickel zinc oxide nanocomposite was prepared using chemical oxidation method. Polyindole and nickel - zinc oxide was also prepared separately to compare their properties with their counterparts.

From SEM analysis it was found that the morphology of $\mathrm{NiO} \& \mathrm{ZnO}$ was changed when they were in composite state. Polyindole showed a cauliflower like pattern which was changed entirely in the case of nanocomposite. From FTIR analysis the vibration bands of all the samples were studied. The phase purity of all the three samples was established by comparison of the X-ray diffraction pattern with JCPDS International data value. From JCPDS card data nickel and nickel - zinc oxide was cubic FCC lattice and zinc was primitive, hexagonal lattice. The lattice constants for all the three samples were calculated and were in agreement with JCPDS Card values. From the crystallite size calculation the average size of the samples were $20-40 \mathrm{~nm}$ respectively. The dielectric studies of allthe samples were done. The capacitance of the polyindole based nickel-zinc oxide nanocomposite was found to be greater than its counter parts. The impedance of these materials was found to be in reverse order which was in agreement with the theory.

Antifungal activity of the nanocomposite and their counterparts were studied separately on the fungi Penicillium Chrysogenum. The nanocomposite showed activity of $1 \mathrm{~cm}$, nickel - zinc oxide showed $0.7 \mathrm{~cm}$ activity, whereas polyindole, nickel oxide and zinc oxide showed no activity. From this study it could be concluded that the nanocomposite could be used as an antifungal material for the fungi Penicillium Chrysogenum.

\section{REFERENCES}

[1] D. Billaud, E. B. Maarouf, and E. Hannecart, Mat.Res.Bull, vol. 29, pp. 1239, 1994.

[2] D. Billaud, E. B. Maarouf, and E. Hannecart, Syn.Met, vol. 69, pp. $571,1995$.

[3] Y. G. Zhang et al., Chem Phys., vol. 20, pp. 601, 2005.

[4] J. Lee, S. Mahendra, and P. Alvarez, ACS Nano., vol. 4, pp. 3580$3590,2010$.

[5] M. A. Karimi et al., Int. Nano Lett., vol. 1, pp. 43-51, 2011.

[6] J. Neamtu and N. Verga, J. of Nanomat. and Biostru., vol. 6, pp. 969978, 2011.

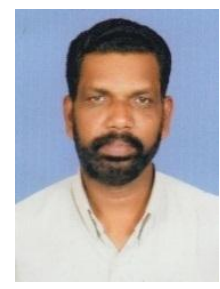

R. Raveendran was born on May 15, 1960 at Kollam, Kerala, India. He graduated in B.Sc physics (1980) from Kerala University and obtained M.Sc in physics (1986) from University of Calicut, Kerala. He obtained Ph.D from M.S. University of Baroda, Gujarat, India, on the topic "Hardness and chemical dissolution of crystalline materials $(\mathrm{NaCl}, \mathrm{KCl}$ and $\mathrm{KBr})$ ". He obtained PDF on the topic "Nanoscience", (2004-2007), University Grand Commission, New Delhi, India.

$\mathrm{He}$ is now teaching physics at various S. N. Colleges in Kerala for the last 23 years and currently working as The Principal, MPMMSN College, Shornur, Kerala, India. He is the research guide of Kerala University, there are five students awarded $\mathrm{Ph} . \mathrm{D}$ under his guidance and many more doing research. He has published around 35 papers in various national and international journals and attended a number of seminars and conference. His research interest included areas of nanoscience, materials science, conducting polymers, etc.

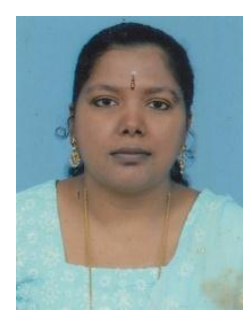

Dedhila Devadathan was born in 1980 at Kollam, Kerala, India. She graduated in B.Sc physics (2001) from Kerala University and obtained M.Sc in Physics (2003) from University of Kerala, India. She obtained M.Phil. from Madurai Kamaraj University, Madurai, Tamil Nadu, India, qualified GATE (2011), qualified NET (June, 2013).

She is now teaching physics at S. N. College, Kollam, Kerala for seven years from 2004 to 2010 She is currently doing research under guidance of $\mathrm{Dr}$. R. Raveendran on the topic "Synthesis, Characterization and applications of Polyindole - metal oxide nanocomposites". She has attended and presented research works in number of national and international seminars and conferences. Her research interest included areas of nanoscience, materials science, conducting polymers, etc. 